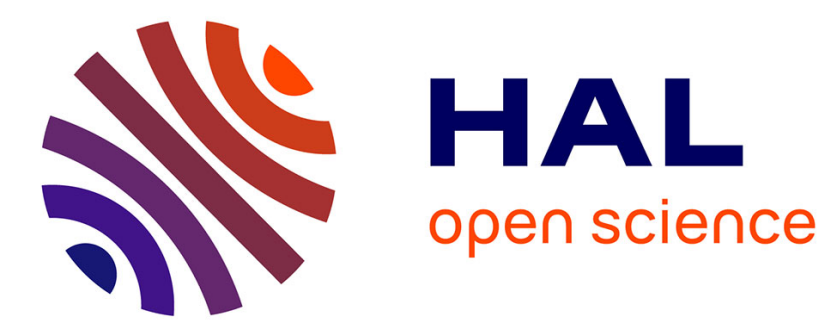

\title{
Bifurcations and Chaos in a Photovoltaic Plant
}

\author{
Mohamed Abdelmoula, B. Robert
}

\section{To cite this version:}

Mohamed Abdelmoula, B. Robert. Bifurcations and Chaos in a Photovoltaic Plant. International journal of bifurcation and chaos in applied sciences and engineering , 2019, 29 (08), pp.1950102. 10.1142/S0218127419501025 . hal-02544861

\section{HAL Id: hal-02544861 \\ https://hal.science/hal-02544861}

Submitted on 16 Apr 2020

HAL is a multi-disciplinary open access archive for the deposit and dissemination of scientific research documents, whether they are published or not. The documents may come from teaching and research institutions in France or abroad, or from public or private research centers.
L'archive ouverte pluridisciplinaire HAL, est destinée au dépôt et à la diffusion de documents scientifiques de niveau recherche, publiés ou non, émanant des établissements d'enseignement et de recherche français ou étrangers, des laboratoires publics ou privés. 


\title{
Bifurcations and Chaos in a Photovoltaic Plant
}

\author{
M. Abdelmoula ${ }^{\mathrm{a}, *}$, B. Robert ${ }^{\mathrm{b}}$ \\ ${ }^{a}$ Département Recherche, Altran France, France \\ ${ }^{b}$ Laboratoire CReSTIC, Université de Reims Champagne-Ardenne, Reims, France
}

\begin{abstract}
This paper presents a comprehensive approach to analyze the dynamics of a photovoltaic system by using discrete-time modeling approach. The proposed structure consists of a photovoltaic array, a two-cell dc-dc buck converter and a load connected in cascade through a dc bus. The research efforts focus on the modeling process and stability analysis, which leads to an implementation with a comprehensive description validated through simulation results. The research efforts focus on the numeric simulation improvements and dynamic investigation of the photovoltaic system under digital controls. The chaotic behaviors that appear in the photovoltaic plant are then studied. The aim of the last part is to detail the stability study using numerical simulation.

Keywords: Photovoltaic array model, Non-causal Modeling, Nonlinear PWM Controller, Two-cell Buck Converter, Discrete-time model, Stability Analysis, Digital Control, Bifurcations, Chaos.
\end{abstract}

\section{Introduction}

Actually, nuclear and fossil fuel are the main energy provider of the worldwide economy. Nevertheless, the recognition of them as being an important cause of environmental degradation makes the humanity to look for alternative resources in power generation. Furthermore, the day-by-day increasing demand for energy can adduce problems for the power distributors, like grid

\footnotetext{
* Corresponding author

Email address: mohamed.abdelmoula@altran.com (M. Abdelmoula )
}

Preprint submitted to International Journal of Bifurcation and Chaos 
instability and even outages. Thence, the necessity of satisfying future energy requirement combined with the interest in clean technologies yields in an increased development of stand-alone plants based on renewable energy systems. Among the renewable energy sources, the photovoltaic generator has one of the largest utilization nowadays. However, due to important characteristics of the photovoltaic sources, power stage systems are usually designed with multiple operating modes. Modeling of these systems is a special challenge. The dynamic behavior is therefore quite complex, and the design for stable operation of such systems requires consideration of the stability conditions for all possible operating modes. This paper describes a stand-alone photovoltaic plant. A mathematical model of this system is developed. Using this model, the system responses to various control parameters will be investigated.

The main purpose of this work is to analyze stability in a photovoltaic plant. The presentation of the work will be divided into three major parts. First, a detailed description of the photovoltaic plant will be presented. The main elements of the photovoltaic system and their characteristics are remembered. A special focus will be done on the photovoltaic array model based on the asymptotic approach. In the second part, a study of new discrete-time modeling approach of the photovoltaic plant will be presented. Numerical simulations and dynamical aspect of the photovoltaic plant are then illustrated in the time domain and in the parameters space. Then, the analysis of some one-dimensional bifurcation diagrams is given. In the third part, we will analyze the stability the new discrete model. Thus, a digital controller will be considered for the system and the conditions of stability are also given. Simulation results illustrate boundaries of stability in the design parameters space will be shown. Finally, the conclusions of this work are summarized.

\section{Design and elementary modeling of the photovoltaic system}

Photovoltaic power generation systems are usually connected to a dc load through a dc-dc power converter. This architecture allows driving of the termi- 
nal voltage of the photovoltaic system in order to manage its power generation under continuously varying operating condition.

The system under consideration in this study is shown in figure $(1)[1,2]$. This structure is based on a dc bus. The photovoltaic array is connected through a two-cell dc-dc buck converter. This structure is intended for applications where the load voltage, (in our case, the output voltage $v_{O}$ ), is lower than the input voltage present at the converter, i.e. the open circuit voltage of the photovoltaic generator. This is the structure of CReSTIC laboratory photovoltaic plant [1]. On the other side, the dc bus is connected to a resistive load. $i_{L}$ is the current on the output terminals of the dc-dc converter. $u_{1}$ and $u_{2}$ are the switched control signals of $S_{1}$ and $S_{2}$ respectively, that can only have the discrete values 0 (switch opened) or 1 (switch closed).

Finally, $C_{1}, C_{2}$, and $L$ are designed to ensure chattering consideration. We assumed that all components of the photovoltaic system are ideal.

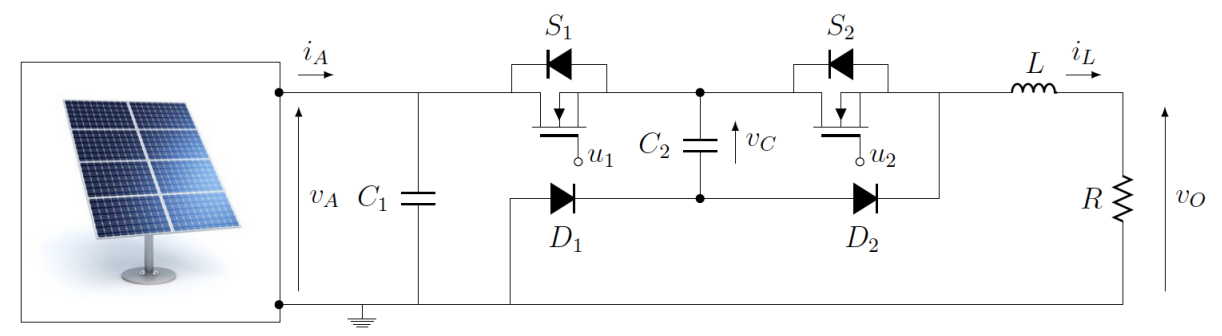

Figure 1: Equivalent circuit of the photovoltaic plant with two-cell buck converter.

\subsection{Photovoltaic Array Modeling}

Several photovoltaic cell models are well discussed in literature using the one-diode and the two diodes photovoltaic cell models $[3,4,5,6,7,8]$. A modified current-voltage relationship which includes the effects of parallel and series connection in photovoltaic array is also described in photovoltaic literature $[9,10,11]$.

In fact, the current-voltage characteristic of the photovoltaic array is crucial in the mathematical analysis problem of the photovoltaic plant model. Hence, 
because of the complexity of the mathematical models which describe this characteristic, it is necessary to simplify it to carry out an analytical stability study task.

In this work, the photovoltaic array is composed of $4 \times 3$ series-parallel Bosch $275 \mathrm{~W}$ modules [1]. The objective here is to present an approximation of the algebraic current-voltage expression of the photovoltaic array in order to reduce the complexity of the numerical analysis.

As shown in figure (2), the current-voltage characteristic curve of the photovoltaic array can be approximated by two asymptotes. They are defined using the photovoltaic array short-circuit current $I_{s c, A}$, the open circuit voltage $V_{o c, A}$, and the maximum power point voltage $V_{m p p}, A \cdot i_{A}$ and $v_{A}$ are the photovoltaic array current and voltage respectively. Using this approximation, the $\left(i_{A}-v_{A}\right)$ characteristic will be defined as:

$$
i_{A}=\left\{\begin{array}{cc}
I_{s c, A} & \text { if } 0 \leq v_{A} \leq V_{m p p, A} \\
-Z_{0}\left(v_{A}-V_{o c, A}\right) & \text { if } V_{m p p, A}<v_{A} \leq V_{o c, A}
\end{array}\right.
$$

Where

$$
Z_{0}=\frac{I_{s c, A}}{V_{o c, A}-V_{m p p, A}}
$$

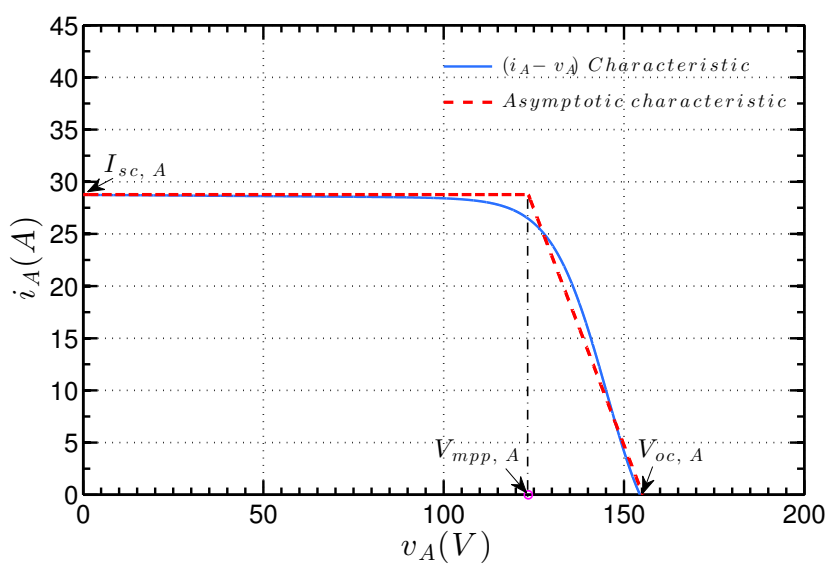

Figure 2: Asymptotic approach of the $\left(i_{A}-v_{A}\right)$ characteristic. 


\subsection{Power Stage System}

As shown in figure (1), the power stage consists of a two-cell dc-dc buck converter. In practice, multicell converter systems are usually chosen for the power stage due to the expected good efficiency in isolated applications [12]. The study of the two-cell dc-dc buck converter has been well developed by Robert et al. in $[13,14,15]$. The use of such converters to drive the output power for a standalone microgrid will provide significant advantages due to their fast response and autonomous control [16]. Under an appropriate control strategy, these converters have a good performance in transient time and in steady state. Regularly, the goal of this control is to balance the flying capacitors voltage $v_{C}$ to a fraction of the input voltage and to adjust the load current to the desired reference level [17].

The signals $u_{1}$ and $u_{2}$ are the output of a digital pulse width modulation (PWM) driven by a feedback control system, which will be designed later. The control signals laws are the switching laws driving $S_{1}$ and $S_{2}$. It should be mentioned that in this work, the duty cycles are defined with respect to the OFF state rather than the ON state. This assumption has not any consequence on the dynamic analysis operation of the buck converter, that's a way of having more simplified models to operate with. Therefore, the switch $S_{k}$ will dwell in its OFF state during $d_{k} T_{s w}$ under the action of the driving signal $u_{k}$ with a duty cycle $d_{k}$. Where $T_{s w}$ is the switching period of the buck converter and $k \in\{1,2\}$. In a continue conduction mode (CCM), because of the natural turn off switching of diodes under negative voltages, the switch $S_{k}$ and the diode $D_{k}$ are always controlled in a complementary way. In other words, under a fixed frequency PWM control, $S_{k}$ is close since $D_{k}$ is open and vice versa. There are two switches, so we can easily distinguish four operating topologies as shown in figure (3).

By applying Kirchhoff laws in the different photovoltaic plant's topologies, we deduce the resulting switched model of the photovoltaic system in CCM is given by (2). 


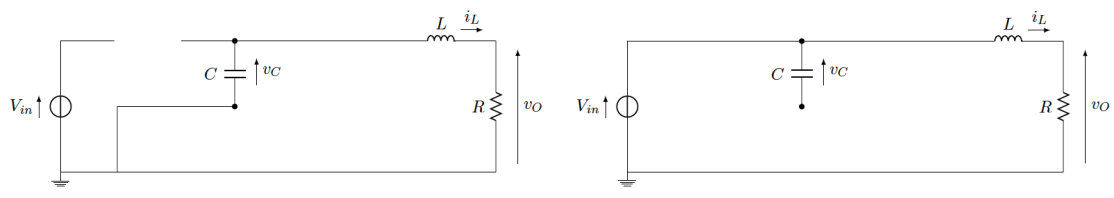

(a) Topology OFF-ON.

(b) Topology ON-ON.

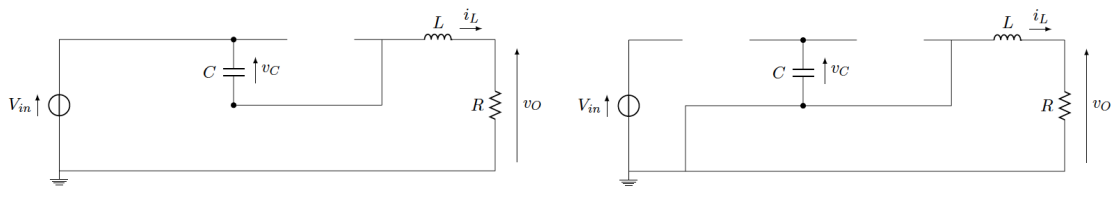

(c) Topology ON-OFF.

(d) Topology OFF-OFF.

Figure 3: Photovoltaic plant topologies.

$$
\left(\begin{array}{c}
\dot{v}_{A} \\
\dot{i}_{L} \\
\dot{v}_{C}
\end{array}\right)=\left(\begin{array}{ccc}
0 & -\frac{1}{C_{1}} u_{1} & 0 \\
\frac{1}{L} u_{1} & \frac{-R}{L} & \left(u_{2}-u_{1}\right) \frac{1}{L} \\
0 & \left(u_{1}-u_{2}\right) \frac{1}{C_{2}} & 0
\end{array}\right)\left(\begin{array}{c}
v_{A} \\
i_{L} \\
v_{C}
\end{array}\right)+\left(\begin{array}{c}
\frac{1}{C_{1}} i_{A} \\
0 \\
0
\end{array}\right)
$$

\section{Discrete-time model of the photovoltaic system}

Usually adopted in the power converter modeling tasks, the linear average model does not make it possible to observe the strange nonlinear phenomena. By its nature, this type of model hides the essential non-linearities of the system by considering its average state on an operating period or by linearizing its dynamics around particular operating points [18]. Thus, to analyze and control these behaviors, we are going to need a non-linear discrete-time model.

Instead of using the exact discrete-time model, Robert et al. propose a new discrete-time modeling approach in order to identify the appearance conditions of unfamiliar running modes $[13,14,19,20]$. This discrete-time modeling approach is based on a stroboscopic sampling of the state variables at the beginning of each switching period $T_{s w}$. The distinctive feature of this discrete-time modeling approach is to demonstrate that a basic model structure shall be sufficient to investigate the real system behaviors properties. In other words, only some 
variables are responsible for the appearance of the systems unnormal running modes [21]. This modeling approach will be adopted for the rest of this work to analytically analyze the non-linear dynamics of the system.

For the interest of reducing the number of parameters of the system, we will consider the dimensionless variables. Hence, the voltages are scaled by the maximum photovoltaic system voltage $V_{\max }$, such as $V_{\max }$ is equal to the photovoltaic array open-circuit voltage $\left(V_{o c, A}\right)$. In addition, the currents are scaled using the maximum photovoltaic system current $I_{\max }$, such as $I_{\max }$ is equal to the photovoltaic array short-circuit current $\left(I_{s c, A}\right)$. Then, the scaled states variables are defined as:

$$
\chi_{v_{A}}(t)=\frac{v_{A}(t)}{V_{\max }}, \chi_{v_{C}}(t)=\frac{v_{C}(t)}{V_{\max }} \text { and } \chi_{i_{A}}(t)=\frac{i_{A}(t)}{I_{\max }}, \chi_{i_{L}}(t)=\frac{i_{L}(t)}{I_{\max }}
$$

By following this modeling approach, and adding the current-voltage photovoltaic array expression defined in (1) to the above switched model (2), the photovoltaic plant discrete-time model is then rearranged to the system of equations expressed in (4).

$$
\begin{aligned}
& \text { - For } 0 \leq v_{A} \leq V_{m p p, A} \\
& \left\{\begin{array}{l}
\chi_{v_{A}}[n+1]=\chi_{v_{A}}[n]-\left(1-d_{1}[n]\right) \delta_{C_{1}} \chi_{i_{L}}[n]+\delta_{C_{1}} \chi_{I_{s c}, A} \\
\chi_{i_{L}}[n+1]=\left(1-d_{1}[n]\right) \delta_{L_{o}} \chi_{v_{A}}[n]+\left(1-\delta_{L}\right) \chi_{i_{L}}[n]+\delta_{d}[n] \delta_{L_{o}} \chi_{v_{C}}[n] \\
\chi_{v_{C}}[n+1]=-\delta_{d}[n] \delta_{C_{2}} \chi_{i_{L}}[n]+\chi_{v_{C}}[n]
\end{array}\right.
\end{aligned}
$$

- For $V_{m p p, A}<v_{A} \leq V_{o c, A}$

$$
\left\{\begin{array}{l}
\chi_{v_{A}}[n+1]=\chi_{v_{A}}[n]-\left(1-d_{1}[n]\right) \delta_{C_{1}} \chi_{i_{L}}[n]-\delta_{C_{1}} \chi_{Z_{0}}\left(\chi_{v_{A}}[n+1]-\chi_{V_{o c}, A}\right) \\
\chi_{i_{L}}[n+1]=\left(1-d_{1}[n]\right) \delta_{L_{o}} \chi_{v_{A}}[n]+\left(1-\delta_{L}\right) \chi_{i_{L}}[n]+\delta_{d}[n] \delta_{L_{o}} \chi_{v_{C}}[n] \\
\chi_{v_{C}}[n+1]=-\delta_{d}[n] \delta_{C_{2}} \chi_{i_{L}}[n]+\chi_{v_{C}}[n]
\end{array}\right.
$$

Where $R_{o}=\frac{V_{\max }}{I_{\max }}, \delta_{C_{1}}=\frac{T_{s w}}{R_{o} C_{1}}, \delta_{C_{2}}=\frac{T_{s w}}{R_{o} C_{2}}, \delta_{L_{o}}=\frac{T_{s w} R_{o}}{L}, \delta_{L}=\frac{T_{s w} R}{L}$, and $\delta_{d}[n]=d_{1}[n]-d_{2}[n]$.

\subsection{Proportional controller}

The controller of a dc-dc power electronics system is commonly based on its small-signal model $[22,23]$. In this section, we apply a proportional controller 
to the photovoltaic system map. Although its simplicity of construction and implementation, the proportional controller should be sufficient to investigate the map properties. Nevertheless, as explained above, unfamiliar behaviors depend only on the system structure and not on the number of secondary parameters [19].

Here, we give expressions of $d_{1}$ and $d_{2}$ that drive the state $\chi_{i_{L}}$ to $I_{r e f}$ and $\chi_{v_{C}}$ to $V_{r e f}$. That is, by defining $\varepsilon_{i}[n]=\chi_{i_{L}}[n]-I_{\text {ref }}$ and $\varepsilon_{v}[n]=\chi_{v_{C}}[n]-V_{\text {ref }}$, the aim of this digital control becomes to drive these error variables to zero. The selected expression of the duty cycles are given by:

$$
\begin{gathered}
d_{1}[n]=k_{i} \varepsilon_{i}[n]+k_{v} \varepsilon_{v}[n] \\
d_{2}[n]=k_{i} \varepsilon_{i}[n]
\end{gathered}
$$

where $k_{i}>0$ and $k_{v}>0$ are the proportional gains of current and voltage errors respectively.

Then, by using equations (5) the closed loop model of the photovoltaic system (4) is given as follows:

- For $0 \leq v_{A} \leq V_{m p p, A}$

$$
\left\{\begin{array}{l}
\chi_{v_{A}}[n+1]=\chi_{v_{A}}[n]-\alpha[n] \delta_{C_{1}} \chi_{i_{L}}[n]+\delta_{C_{1}} \chi_{I_{s c}, A} \\
\chi_{i_{L}}[n+1]=\alpha[n] \delta_{L_{o}} \chi_{v_{A}}[n]+\left(1-\delta_{L}\right) \chi_{i_{L}}[n]+k_{v} \delta_{L_{o}} \varepsilon_{v}[n] \chi_{v_{C}}[n] \\
\chi_{v_{C}}[n+1]=-k_{v} \delta_{C_{2}} \varepsilon_{v}[n] \chi_{i_{L}}[n]+\chi_{v_{C}}[n]
\end{array}\right.
$$

- For $V_{m p p, A}<v_{A} \leq V_{o c, A}$

$$
\left\{\begin{array}{l}
\chi_{v_{A}}[n+1]=\frac{1}{1+\delta_{C_{1}} \chi_{Y_{0}}}\left(\chi_{v_{A}}[n]-\delta_{C_{1}} \alpha[n] \chi_{i_{L}}[n]+\delta_{C_{1}} \chi_{Y_{0}} \chi_{V_{o c}, A}\right) \\
\chi_{i_{L}}[n+1]=\alpha[n] \delta_{L_{o}} \chi_{v_{A}}[n]+\left(1-\delta_{L}\right) \chi_{i_{L}}[n]+k_{v} \delta_{L_{o}} \varepsilon_{v}[n] \chi_{v_{C}}[n] \\
\chi_{v_{C}}[n+1]=-k_{v} \delta_{C_{2}} \varepsilon_{v}[n] \chi_{i_{L}}[n]+\chi_{v_{C}}[n]
\end{array}\right.
$$

where $\alpha[n]=1-k_{i} \varepsilon_{i}[n]-k_{v} \varepsilon_{v}[n]$

In the next paragraph this discrete-time model, sampled at the switching frequency, will be used to perform the simulation results of the photovoltaic system. 


\subsection{Simulation of the discrete-time model}

Photovoltaic plant discrete-time model (6) presents two important advantages. First, this model is unique for all operating modes of the CCM. Second, the model simplicity facilitates the analytical analysis procedure. Note that from duty cycles expressions $d_{1}[n]$ and $d_{2}[n]$ can be bigger than the unity or negative which has not practical sense. In order to overcome this problem, the duty cycle will be bounded due to the use of a saturation function sat $\left(d_{k}[n]\right)$ given by (7) and depicted in figure (4) [15]. Thus, the applied duty cycle in (6) is in fact sat $\left(d_{k}[n]\right)$ and not $d_{k}[n]$.

$$
\operatorname{sat}\left(d_{k}[n]\right)=\frac{1}{2}\left(1+\left|d_{k}[n]\right|-\left|d_{k}[n]-1\right|\right)
$$

It has to be pointed out that if the power stage switching period $\left(T_{s w}\right)$ is much

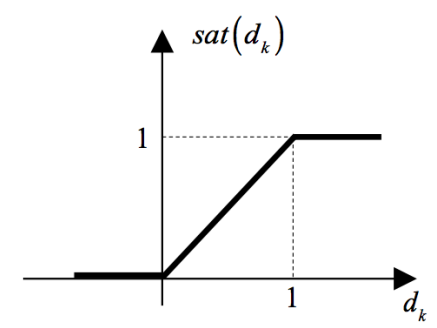

Figure 4: Duty cycle saturation function.

smaller than the main time constant of the load, the current ripple, as well as the voltage ripple will be minimal. In fact, in order to reduce current and voltage ripple through the photovoltaic plant components, the circuit parameters should satisfy $\delta_{L} \ll 1, \delta_{L_{o}} \ll 1$ and $\delta_{C_{i}} \ll 1(i=\{1,2\})$.

The parameters used for the following simulation are: $\delta_{C_{1}}=\delta_{C_{2}}=0.1$ and $\delta_{L_{o}}=\delta_{L}=0.1$. The reference current is arbitrary chosen as $I_{r e f}=0.6$, and since it is required that $v_{C} \simeq \frac{v_{A}}{2}, V_{\text {ref }}$ is fixed to 0.5 .

Figure (5) depicts the response of the variables $\chi_{v_{A}}, \chi_{i_{A}}, \chi_{v_{C}}$ and $\chi_{i_{L}}$ of the photovoltaic plant in closed-loop to an impulse change in $d_{1}$ and $d_{2}$. The selected proportional gains are $k_{i}=9$ and $k_{v}=10$. As we can see, it is a 1T-periodic 


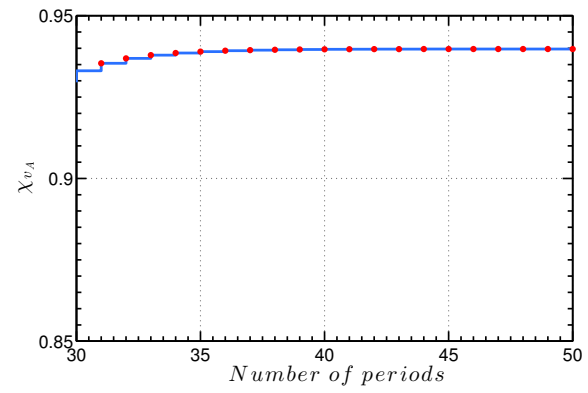

(a) Scaled photovoltaic array voltage $\chi_{v_{A}}$.

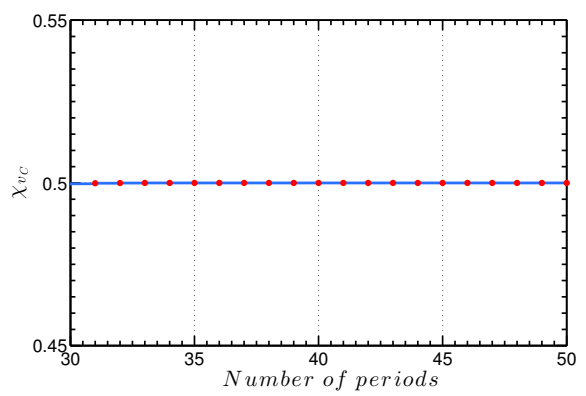

(c) Scaled output capacitor voltage $\chi_{v_{C}}$.

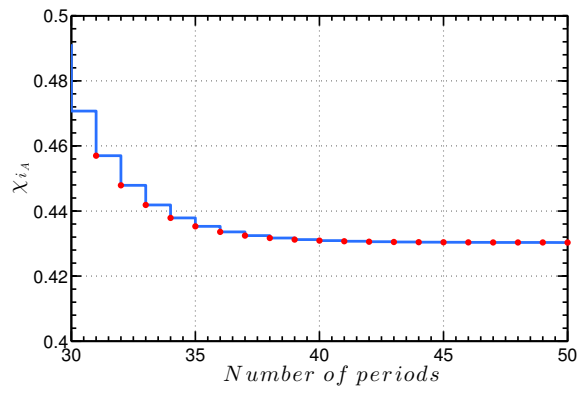

(b) Scaled photovoltaic array current $\chi_{i_{A}}$.

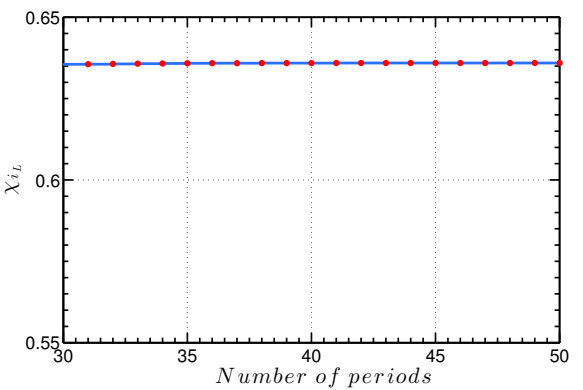

(d) Scaled inductor current $\chi_{i_{L}}$.

Figure 5: Convergence towards the fixed point under action of proportional controller $\left(k_{i}=9, k_{v}=10\right)$.

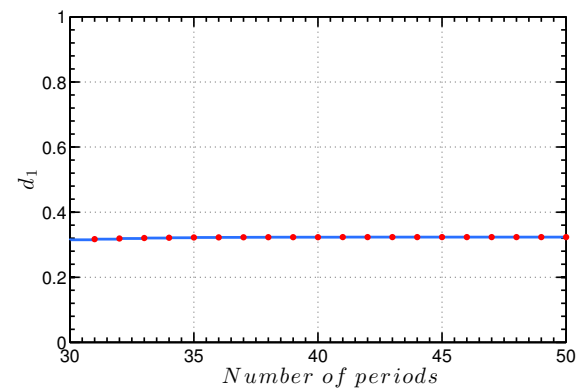

(a) Duty cycle $d_{1}$.

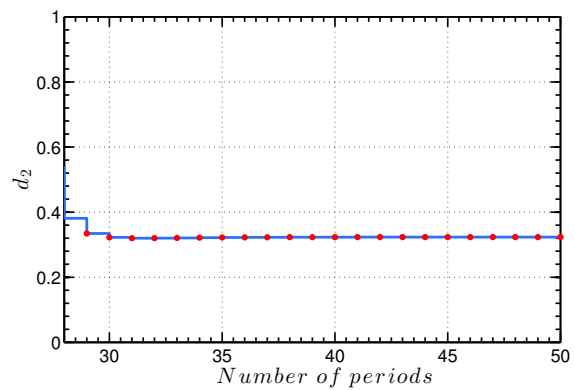

(b) Duty cycle $d_{2}$.

Figure 6: Convergence towards the fixed point of the duty cycles $d_{1}$ and $d_{2}\left(k_{i}=9\right.$, $\left.k_{v}=10\right)$. 
behaviour. On the one hand, there is an exact convergence of $\chi_{v_{C}}$ to $V_{\text {ref }}$, see figure $(5(\mathrm{c}))$. On the other hand, at steady state, we note that $\chi_{i_{L}}=0.64$. So, a non-zero static error is obtained of the inductor current, see figure $(5(d))$. Moreover, as shown in figure (6), at steady state we have $d_{1}=d_{2}=0.32$. To drop the static current error we should progressively increase the current gain $k_{i}$. Then we obtain complex behavior, in the sense of engineering, as shown in figure (7). This operating behavior occurs after losing stability of the 1T-

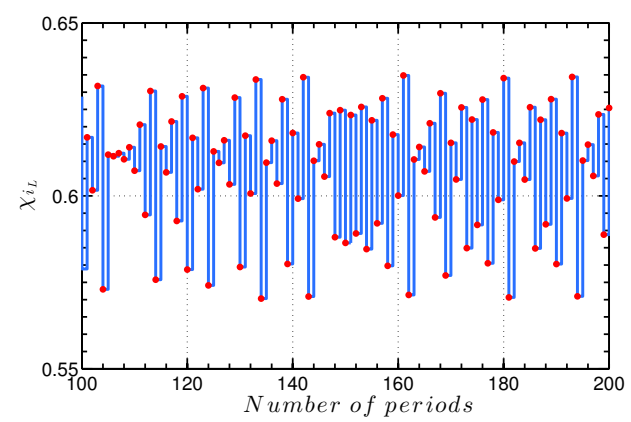

Figure 7: Scaled inductor current $\chi_{i_{L}}$ evolution under action of proportional controller $\left(k_{i}=30, k_{v}=10\right)$.

periodic behavior due to the important value of current gain $k_{i}$.

In the following paragraph, we will compute and analyze bifurcation diagrams for the photovoltaic plant controlled by the previous described proportional controller.

\subsection{One-dimensional bifurcations diagrams: $k_{i}$ as bifurcation parameter}

Bifurcation diagrams illustrate the variations in the photovoltaic system behavior when a parameter is varied. All the diagrams that will be shown later were built with the same simulation method based on the discrete photovoltaic model (6). The bifurcation diagrams are obtained under variation of $k_{i}$. We will clarify the effects of the variation of the current gain on the stability of the photovoltaic system at Standard Test Conditions (STC) $\left(T=25^{\circ} \mathrm{C}, \mathrm{G}=1000 \mathrm{~W} / \mathrm{m}^{2}\right)$. All the diagrams obtained below were built keeping constant these parameters: 
$\delta_{C 1}=\delta_{C 2}=0.1, \delta_{L_{o}}=\delta_{L}=0.1, I_{\text {ref }}=0.6, V_{\text {ref }}=0.5$, and $R=R_{o}$. The initial conditions where fixed at zero [24]. The control parameter $k_{i}$ is varied in the range $[10,30]$ while $k_{v}$ is fixed to 10 . Figures $(8-11)$ show the bifurcation thus obtained.

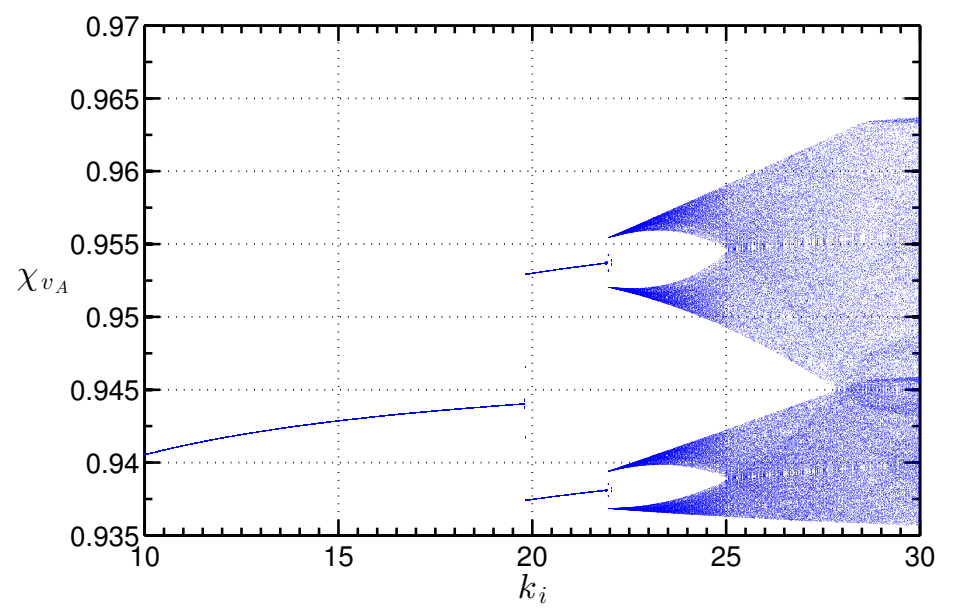

Figure 8: Bifurcation diagram of $\chi_{v_{A}}$ with respect to $k_{i}$.

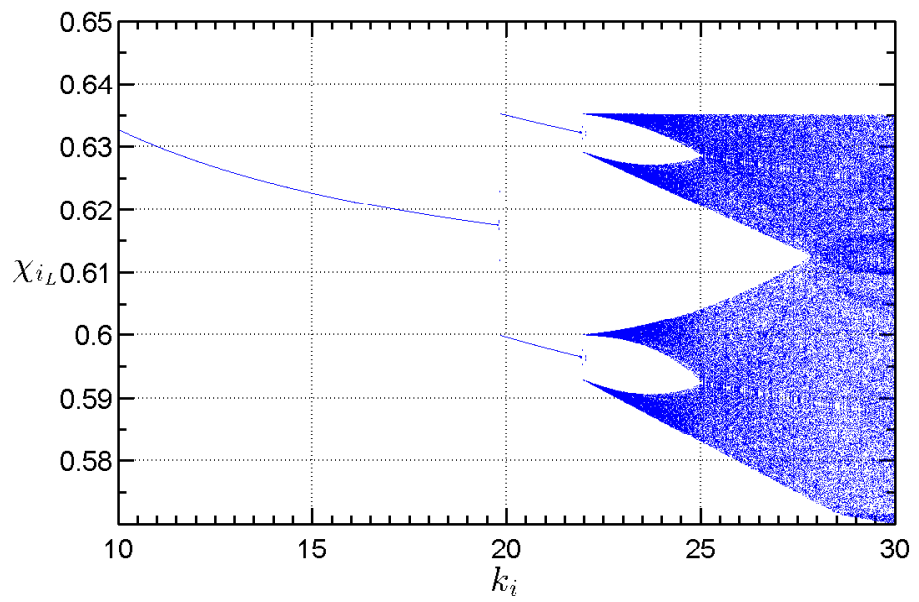

Figure 9: Bifurcation diagram of $\chi_{i_{L}}$ with respect to $k_{i}$.

According to the diagrams depicted in figures (8), (9) and (11), the bifurca- 


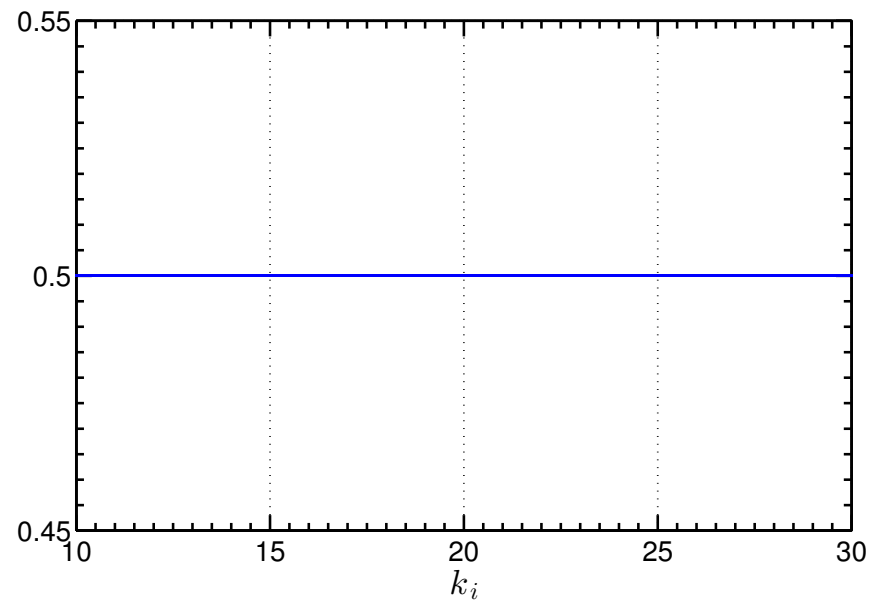

Figure 10: Bifurcation diagram of $\chi_{v_{C}}$ with respect to $k_{i}$.

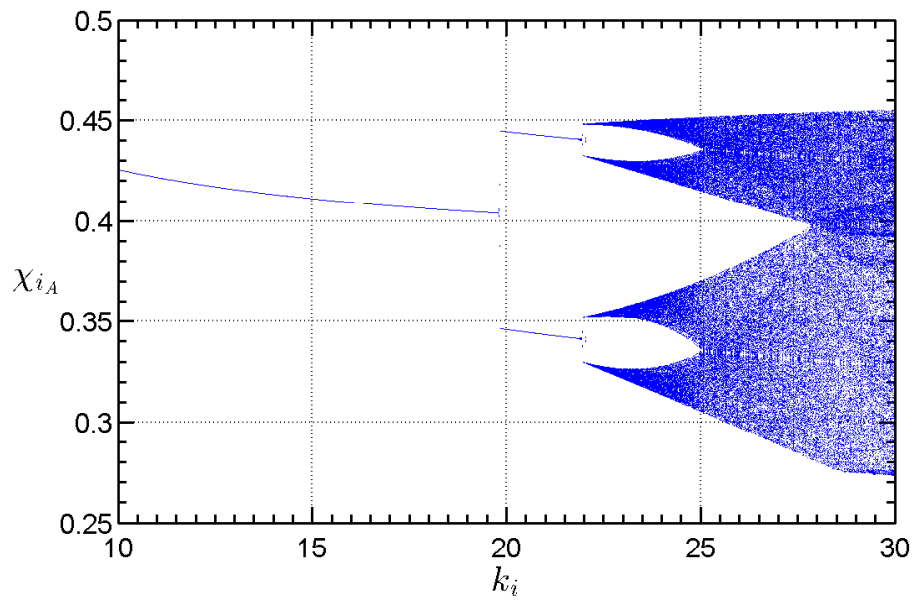

Figure 11: Bifurcation diagram of $\chi_{i_{A}}$ with respect to $k_{i}$.

tion of the solution from period one to period two occurs when $k_{i, \text { crit }} \simeq 19.8$ for $\chi_{v_{A}}, \chi_{i_{L}}$, and $\chi_{i_{A}}$. For values of the current gain $k_{i}$ greater than this critical value, a $2 \mathrm{~T}$-periodic behavior appears and beyond another bifurcation a chaotic behavior takes place. It is also worth to note that beyond the bifurcation value of $k_{i}$, as well as above, there is exact convergence of $\chi_{v_{C}}$ to $V_{\text {ref }}$ (see figure 10). 


\subsection{Chaos detection and Lyapunov exponents}

As we know, trajectories of chaotic system diverge exponentially. The exponential rate divergence between trajectories is one of the measures that characterize the chaotic behavior and detect it. It conditions the ability to predict the system behavior of more or less long-term.

It is common to read that the non-periodic motions (the blue areas) depicted in figures (8), (9) and (11) are chaotic mentions. However, it necessary to even proves it because the quasi-periodic motions have also the same appearance. To characterize non-periodic behaviors, many tools are detailed in literature like fractal dimensions and Lyapunov exponents [25]. By definition, the Lyapunov exponents associated with a trajectory are basically a measure of the rates of expansion and contraction of trajectory neighboring it [26]. They describe the exponential rate at which a tiny perturbation to a trajectory grows or decays in the course of time evolution in the state space. Thus, they are directly related to the system sensitivity to initial conditions.

In consideration of the Lyapunov exponents, a chaotic attractor is characterized by the existence of at least one positive exponent, a zero exponent, and all other negative exponents [27]. In addition, the stability of chaotic dynamics, in other words, the existence of a chaotic attractor set, requires that the sum of exponents is negative. Therefore, we can deduce that the minimum state space dimension allowing the existence of a chaotic solution, to an autonomous continuous-time, is equal to three. Furthermore, using Lyapunov exponents, we can distinguish between different dynamical systems solutions motions.

Several discrete and continuous methods for computing the Lyapunov exponents of dynamical systems are detailed in literature [28]. The most popular methods are based on the singular values or the $Q R$ decomposition [29]. In recent years, significant efforts have been produced to improve the accuracy of $Q R$ orthonormalization techniques, on which strongly depends the estimation of Lyapunov exponents [30]. Along this line, from several orthonormalization methods, we chose the classic Gram-Schmidt method [31, 32]

The number of Lyapunov exponents for a system is equal to the dimension of 
its phase space. Normally the largest exponent designated by $\lambda$ is used because it determines the horizon of the predictability of the system and shows the separation rate of the dynamics trajectories infinitesimally close. In this case, the prediction of the behavior of the system is possible and is based on its sign: - $\lambda<0$ : stable system. The more negative the exponent, the faster the stability. - $\lambda=0$ : stable system in limit cycle.

- $\lambda>0$ : unstable system or chaotic system. Here, we have computed the
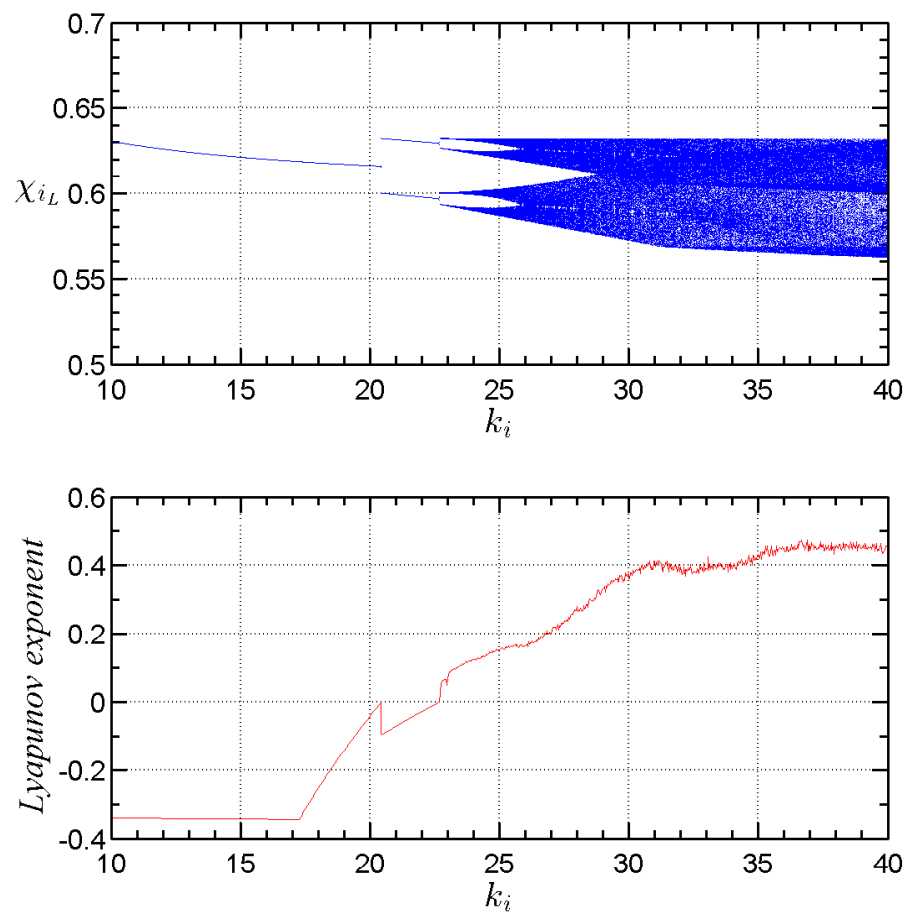

Figure 12: Lyapunov exponent and bifurcation diagram of $\chi_{i_{L}}$ with respect to $k_{i}$.

Lyapunov exponent using the Gram-Schmidt algorithm. The aim of calculating the Lyapunov exponent here is principal to verify quasi-periodicity and chaos that has been predicted from the bifurcation diagram. The largest Lyapunov exponent, as well as the bifurcation diagram of the logistic map, are shown in figure (12). For this particular case, the map exhibits quasi-periodicity at some particular values of the bifurcation parameter. It can also exhibit chaos 
in the region beyond $k_{i} \simeq 22.7$. As previously mentioned, negatives values of Lyapunov exponents implies the stability of periodic motions.

Usually, a classic study of the dynamical system should stop at this level of analysis. The aim here is to continue the dynamic investigation and the stability analysis of the photovoltaic system.

\section{Stability Analysis}

Given that the effect of increasing the current gain $k_{i}$ may lead to bifurcations. In this section, we will analyze the stability of the photovoltaic discrete model. Thus, the conditions of stability are given.

\subsection{Fixed Points Existence}

The 1T-periodic operating mode of the photovoltaic plant under the designed proportional controller corresponds to the equilibrium points of the map (6). These points denoted by $\chi^{*}=\left[\chi_{v_{A}}^{*}, \chi_{i_{L}}^{*}, \chi_{v_{C}}^{*}\right]^{\prime}$, are obtained by solving:

$$
\begin{aligned}
& \chi_{v_{A}}^{*}[n+1]=\chi_{v_{A}}^{*}[n] \\
& \chi_{i_{L}}^{*}[n+1]=\chi_{i_{L}}^{*}[n] \\
& \chi_{v_{C}}^{*}[n+1]=\chi_{v_{C}}^{*}[n]
\end{aligned}
$$

We solved analytically the third equations of maps (6a) and (6b), which are independent of the first two equations. The resolution led to the unique solution $\chi_{v_{C}}^{*}=V_{\text {ref }}$.

The analytical resolution of the first two equations of (8) was performed using the formal computational functions of MATLAB. It leads to two solutions for $v_{A} \in\left[0, V_{m p p, A}\right]$ and three solutions for $\left.\left.v_{A} \in\right] V_{m p p, A}, V_{o c, A}\right]$. The resulting analytical solutions are too complex, we will not give them here. These expressions are used to plot the evolution of fixed points in different planes. Note that these graphs are not the result of a numerical simulation of the fixed points, but rather of the calculation of analytic expressions of fixed points.

To discuss the physical validity of the solutions we assume here that the state variables remain inferior or equal to one. It is also worth to note that if 
we deviate from the STC operating condition, some states variables can exceed the value one, in fact, this analysis also holds outside STC.

Since $\chi_{v_{C}}^{*}=V_{r e f}$, then duty cycles expressions (5a) and (5b) lead to:

$$
d_{1}[n]=d_{2}[n]
$$

Thence, both duty cycles have similar upper and lower saturation limits. These boundaries occur when the duty cycles $d_{k}$ is equal to 1 or 0 . It yields to:

$$
\begin{gathered}
\text { If } d_{k}[n]=1 \text { then } \chi_{i_{L}, \text { sat, upper }}=\frac{1}{k_{i}}+I_{\text {ref }} \\
\text { If } d_{k}[n]=0 \text { then } \chi_{i_{L}, \text { sat, lower }}=I_{\text {ref }}
\end{gathered}
$$

We note that, when $k_{i} \rightarrow \infty$, the boundary $d_{k}[n]=0$ is never crossed. Since, boundaries expressions are independent of $\chi_{v_{A}}$, only $\chi_{i_{L}}$ is studied.

The existence conditions of a fixed point can then be described as:

$$
\chi_{i_{L, \text { sat }, \text { lower }}}<\chi_{i_{L}}^{*}<\chi_{i_{L, \text { sat }, \text { upper }}}
$$

- For $0 \leq v_{A} \leq V_{m p p, A}$

Here, we distinguish two solutions that are real for $\left.\left.k_{i} \in\right] 0 k_{i, 1}\right] \cup\left[k_{i, 2} \infty[\right.$, where $k_{i, 1}$ and $k_{i, 2}$ are equal to 0.37 and 7.40 respectively. However, only one of the two computed solutions satisfies the previous detailed modeling assumptions $\left(\chi_{i_{L}} \leq 1\right)$. In addition, we note that for $0<k_{i} \leq k_{i, 1}$, very high values of $\chi_{i_{L}}^{*}$ are observed. Then only values of $k_{i}$ greater than $k_{i, 2}$ are used later.

Figure (13) presents the evolution of $\chi_{i_{L}}^{*}$ where $k_{i}$ ranges between $k_{i, 2}$ and 40. The curves in red represent the upper and the lower saturation boundaries respectively. As we can see in figure (13), the fixed point (the blue line) never visits the area of existence (the green area of the $\left(k_{i}, \chi_{i_{L}}^{*}\right)$ plane). Since the conditions of existence are not satisfied, the fixed point of the map (6a), does not really exist. Therefore, there is no equilibrium point in the range $\left[0, V_{m p p} A\right]$.

- For $V_{m p p, A}<v_{A} \leq V_{o c, A}$

Here, equation (8) leads to a cubic equation on $\chi_{i_{L}}^{*}$. This equation has one real root and two non-real complex conjugate roots for each $k_{i}>0$. 


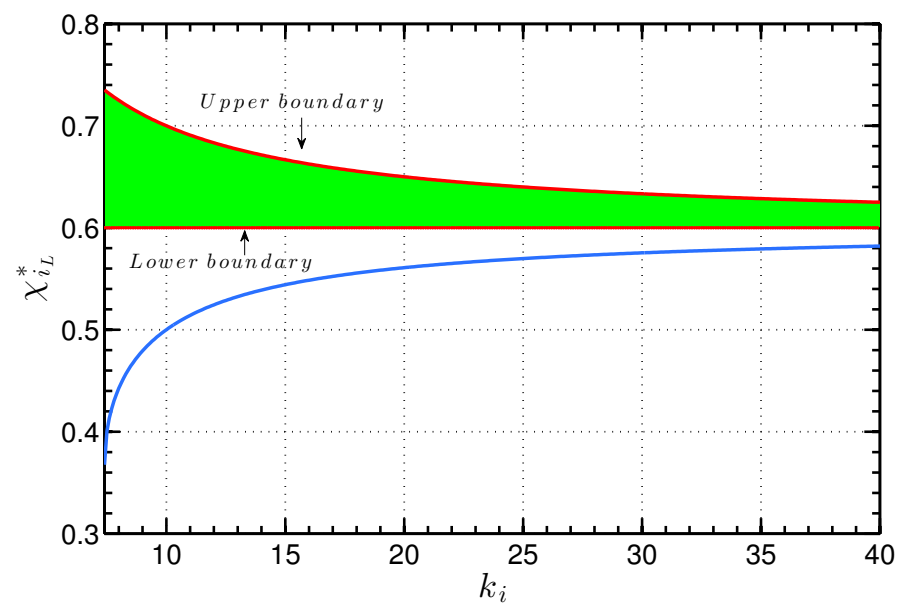

Figure 13: Evolution of $\chi_{i_{L}}^{*}$ in depend of $k_{i}$ for map (6a).

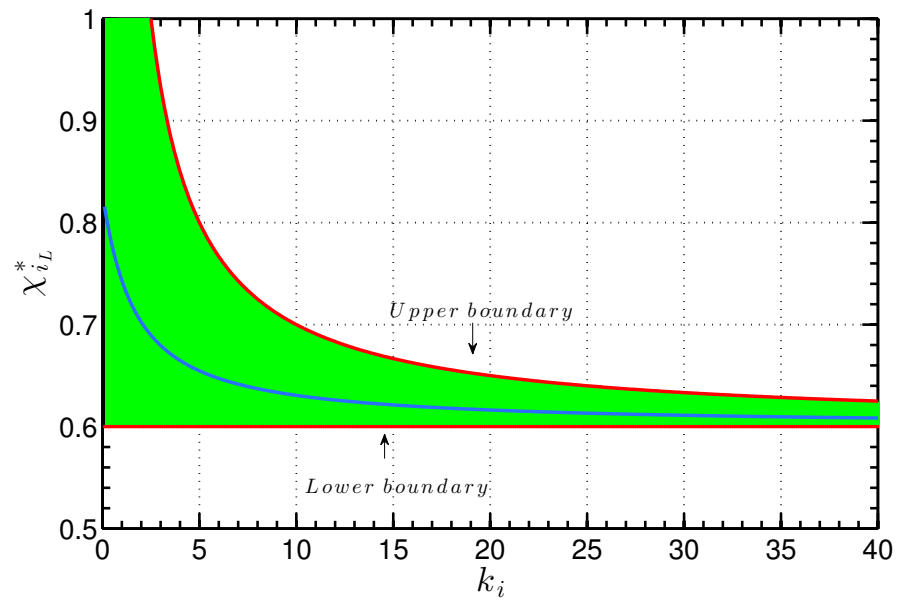

Figure 14: Evolution of $\chi_{i_{L}}^{*}$ in depend of $k_{i}$ for map (6b).

Contrary to the previous map, here the existence conditions are satisfied for each $k_{i}$. We note from figure (14), that $\chi_{i_{L}}^{*}$, don't never leaves the existence region. We conclude that the photovoltaic plant has a unique fixed point located in the interval $\left[V_{m p p}, A, V_{o c, A}\right]$. Then the following stability study is carried out only for $V_{m p p, A}<v_{A} \leq V_{o c, A}$, i.e. only for the map (6b). 


\subsection{Fixed Points Stability}

Based on the expression of the Jacobian matrix we can study the evolution of its eigenvalues as $k_{i}$ and $k_{v}$ are varied. The Jacobian matrix of the map (6b) is given as follows:

$$
J_{b}=\left[\begin{array}{ccc}
\frac{\partial \chi_{v_{A}}[n+1]}{\partial \chi_{v_{A}}[n]} & \frac{\partial \chi_{v_{A}}[n+1]}{\partial \chi_{i_{L}}[n]} & \frac{\partial \chi_{v_{A}}[n+1]}{\partial \chi_{v_{C}}[n]} \\
\frac{\partial \chi_{i_{L}}[n+1]}{\partial \chi_{v_{A}}[n]} & \frac{\partial \chi_{i_{L}}[n+1]}{\partial \chi_{i}[n]} & \frac{\partial \chi_{i_{L}}[n+1]}{\partial \chi_{v_{C}}[n]} \\
\frac{\partial \chi_{v_{C}}[n+1]}{\partial \chi_{v_{A}}[n]} & \frac{\partial \chi_{v_{C}}[n+1]}{\partial \chi_{i_{L}}[n]} & \frac{\partial \chi_{v_{C}}[n+1]}{\partial \chi_{v_{C}}[n]}
\end{array}\right]
$$

Stability is ensured if all eigenvalues are inside the unit circle in the complex plane. Linearizing system (6b) around its fixed point leads to the following Jacobian matrix:

$$
J_{b}\left(\chi^{*}\right)=\left[\begin{array}{ccc}
J_{b, 11} & J_{b, 12} & J_{b, 13} \\
J_{b, 21} & J_{b, 22} & J_{b, 23} \\
0 & 0 & J_{b, 33}
\end{array}\right]
$$

where

$$
\begin{aligned}
J_{b, 11} & =\frac{1}{1+\delta_{C_{1}} \chi_{Y_{0}}} \\
J_{b, 12} & =\frac{\delta_{C_{1}}\left(2 k_{i} \chi_{i_{L}}^{*}-1-k_{i} I_{r e f}\right)}{1+\delta_{C_{1}} \chi Y_{0}} \\
J_{b, 13} & =\frac{k_{v} \delta_{C_{1}} \chi_{i_{L}}^{*}}{1+\delta_{C_{1}} \chi_{0}} \\
J_{b, 21} & =\left(1-k_{i} \delta_{i_{L}}\right) \delta_{L_{o}} \\
J_{b, 22} & =-k_{i} \delta_{L_{o}} \chi_{v_{A}}^{*}+\left(1-\delta_{L}\right) \\
J_{b, 23} & =\delta_{L_{o}} k_{v}\left(V_{r e f}-\chi_{v_{A}}^{*}\right) \\
J_{b, 33} & =-k_{v} \delta_{C_{2}} \chi_{i_{L}}^{*}+1
\end{aligned}
$$

where $\delta_{i_{L}}=\chi_{i_{L}}^{*}-I_{\text {ref }}$.

Figure (15) shows the loci of the eigenvalues when $k_{i}$ varies for different values of $k_{v}$.

For small values of $k_{i}$, the first two eigenvalues $\lambda_{1}$ and $\lambda_{2}$ are complex conjugate, then by increasing $k_{i}$ they become real. In addition $\lambda_{1}$ don't leaves the unit circle. Contrary to $\lambda_{1}$, for large values of $k_{i}, \lambda_{2}$ leaves the circle unit by 

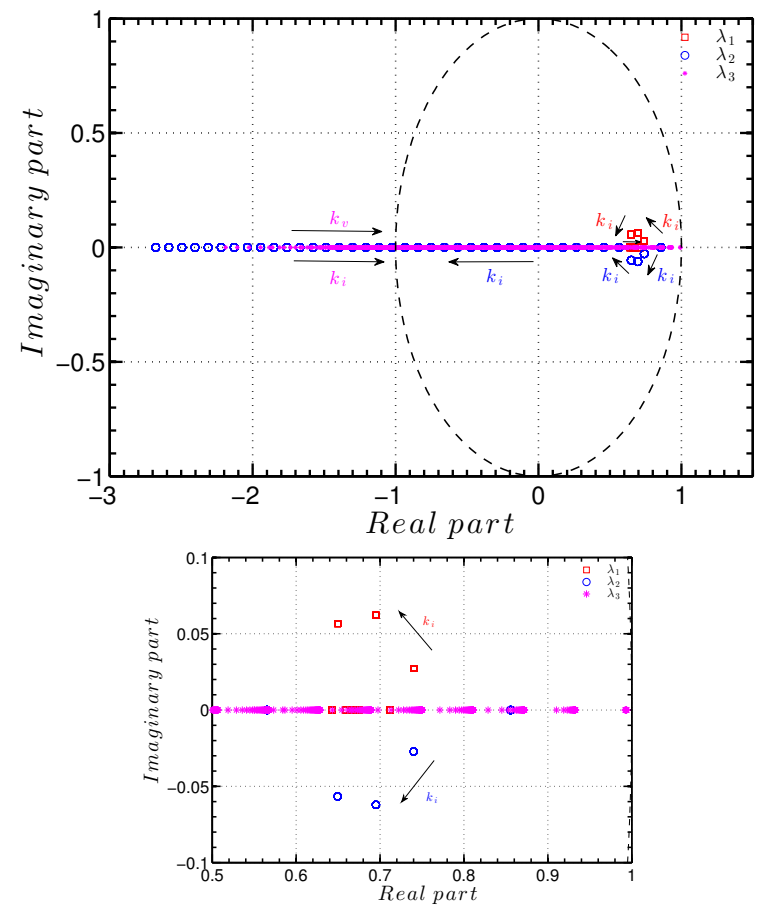

Figure 15: Evolution of the eigenvalues of the Jacobian matrix when $k_{i}$ are $k_{v}$ are varied for map (6b).

the real point $(-1)$. Moreover, $\lambda_{1}$ and $\lambda_{2}$ are independent of $k_{v}$. We note that there are two eigenvalues which can destabilize the fixed point: $\lambda_{2}$ when $k_{i}$ is too large, and $\lambda_{3}$ when $k_{i}$ is too small.

The examination of the eigenvalues loci shows that it is necessary to analyze the stability boundaries.

\subsection{Stability boundaries}

The loss of stability in a neighborhood of a fixed point can be studied through the linear approximation of the discrete system, that is the well known Taylor expansion truncated after the first order terms. In practice, the cubic characteristic polynomials resulting from the photovoltaic system tend to be tediously complicated. The analytic study of the characteristic polynomial is carried out in the same way as the analytic search of the fixed points. Thus, the expres- 
sions of the fixed points are injected into the elements of the Jacobian (14) and the eigenvalues are computed using a formal calculation tool [33]. The expression of the eigenvalues can then be evaluated in the parameter plane $\left(k_{i}, k_{v}\right)$. This method help in determining the stability regions of the fixed points. The boundaries stability can be plotted in a suitable parameters space.

To ensure stability, the eigenvalues of the system should be placed inside the unit circle of the complex plane by tuning the control gain $k_{i}$ and $k_{v}$. Figures (16-18) show the optimal values of the pair $\left\{k_{i}, k_{v}\right\}$ necessary to minimize the modulus of eigenvalues $\lambda_{1}, \lambda_{2}$, and $\lambda_{3}$ of the Jacobian (14).

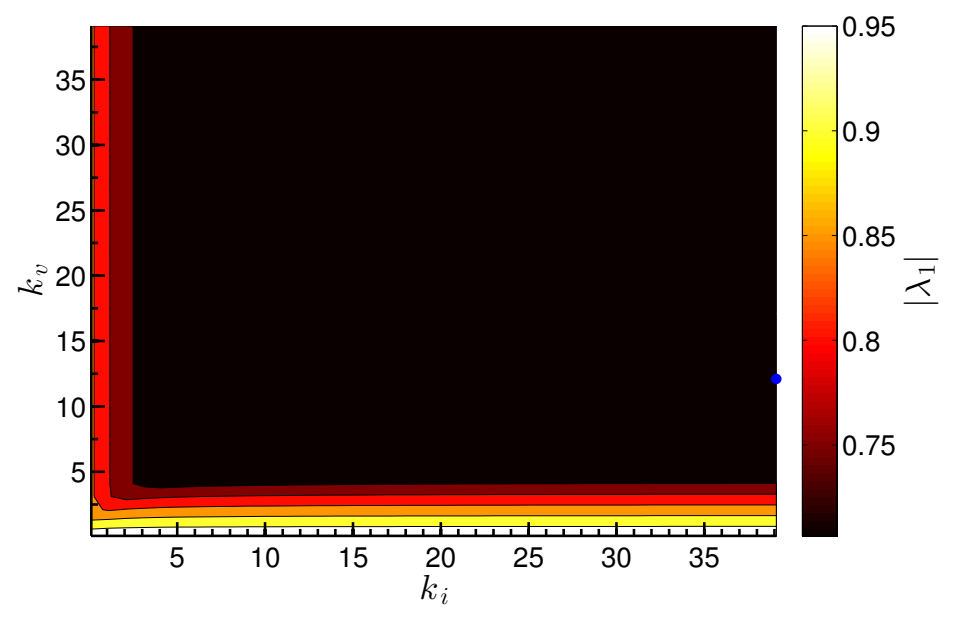

Figure 16: The stability zone of $\lambda_{1}$ in the parameter plane $k_{i}-k_{v}$.

As may be seen, the boundary limits are depicted by one-level lines. These lines present the set of critical values of gains pair $\left\{k_{i, c r i t}, k_{v, \text { crit }}\right\}$. It can be observed that as $k_{i}$ and $k_{v}$ increase, eigenvalues cross the one-level lines, leaving the stability zone, indicating that a bifurcation occurs at a critical pair $\left\{k_{i, c r i t}, k_{v}\right.$, crit $\}$, see figures (17) and (18). The fixed point becomes nonhyperbolic, that is the modulus of complex conjugates pair of eigenvalues or real eigenvalue is equal to 1 . Thence, it may be deduced that the boundary of stability of the photovoltaic system is given by the loci of the third eigenvalue $\lambda_{3}$ of the Jacobian matrix $J_{b}\left(\chi^{*}\right)$. Hence, the stable region of the system is de- 


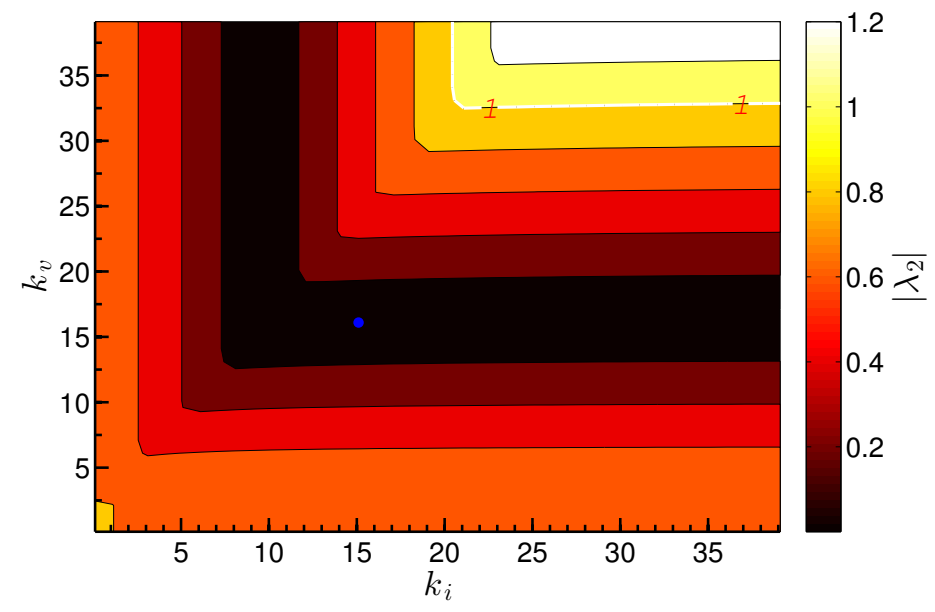

Figure 17: The stability zone of $\lambda_{2}$ in the parameter plane $k_{i}-k_{v}$.

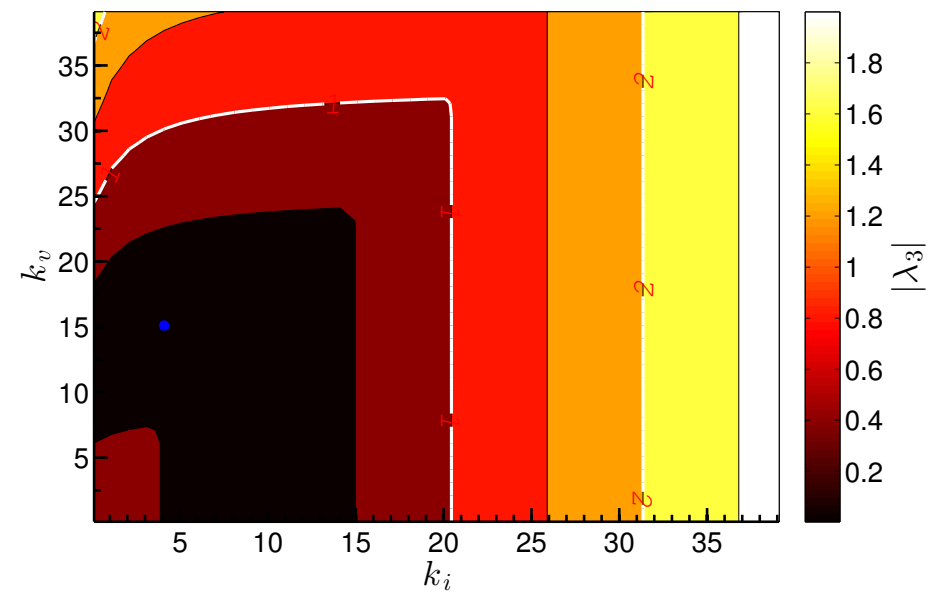

Figure 18: The stability zone of $\lambda_{3}$ in the parameter plane $k_{i}-k_{v}$.

picted in figure (18). To obtain a fast response, we may impose the eigenvalues as close as possible to the origin of the unit circle. This can be achieved by selecting the pair $\left\{k_{i}, k_{v}\right\}$ which correspond to the minimum of the eigenvalue modulus, presented in figure (18) by a blue points. The optimal pairs values $\left\{k_{i, \text { opt }}, k_{v, \text { opt }}\right\}$, are represented by blue point. 
Figure (19) depicts the effect of the proportional controller with $k_{i}=15$, where $k_{v}$ is fixed to 18.1. As it was observed in figure section (3), we distinguish that a non-zero static error is obtained for the inductor current, see figure (19(a)). We showed previously that to reduce the static error, we must increase the current gain, which leads to the loss of stability. In the same context, figure (20) delineates the asymptotic response of the system to the proportional controller with $k_{i}=35$ and $k_{v}=18.1$. As shown in figure (20(a)), $\chi_{i_{L}}$ presents a chaotic behavior. Note, that the duty cycles undergo saturations, see figures (20(b)) and (20(c)). The saturation function is strongly nonlinear. It is the main cause of the onset of chaos. In fact, we know that chaos does not appear in the linear systems, but we have studied a linearized model. Since we observe a chaotic behavior, it is due to the control non-linearity.

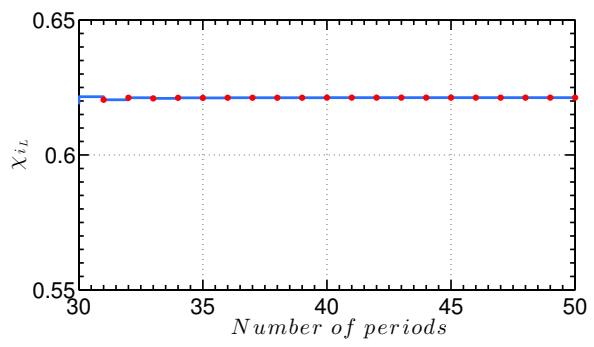

(a) Scaled inductor current $\chi_{i_{L}}$.

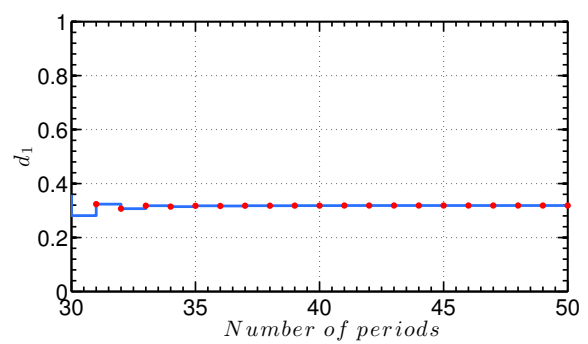

(b) Duty cycle $d_{1}$.

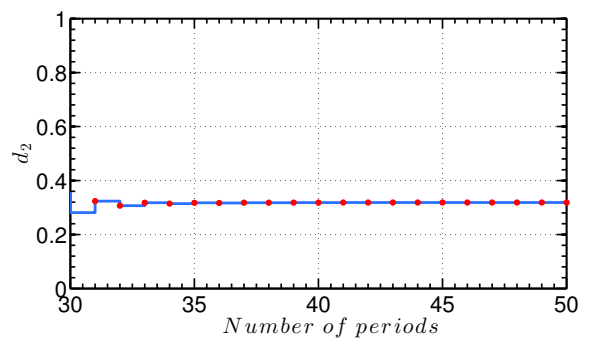

(c) Duty cycle $d_{2}$.

Figure 19: Time evolution under action of proportional controller $\left(k_{i}=15, k_{v}=18.1\right)$.

The bifurcation diagram of figure (21) gives an overview of the global behavior of the photovoltaic system when the current gain $k_{i}$ is varied. The current 


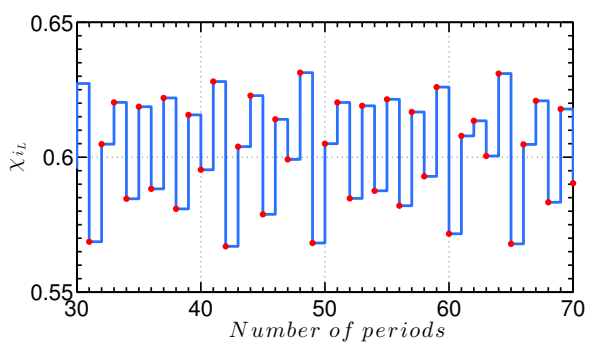

(a) Scaled inductor current $\chi_{i_{L}}$.

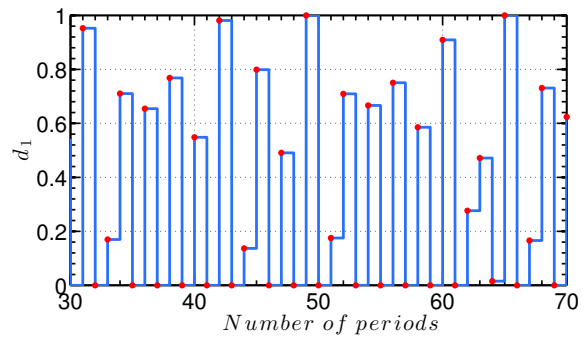

(b) Duty cycle $d_{1}$.

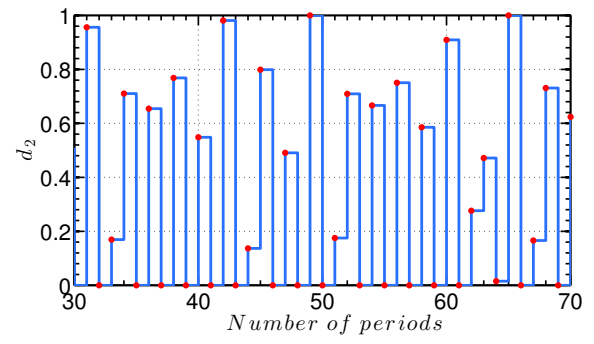

(c) Duty cycle $d_{2}$.

Figure 20: Time evolution under action of proportional controller $\left(k_{i}=35, k_{v}=18.1\right)$.

reference is fixed to $I_{r e f}=0.6$ and the voltage gain $k_{v}=18.1$. We note that, on the left side, the normal fixed point is moving towards the reference when $k_{i}$ increases. Beyond $k_{i}=20.8$, a stable two periodic running mode appears, as a result of a flip bifurcation. Then the current alternates, first inside two chaotic strips, and finally in a unique chaotic set. The Flip bifurcation occurs when one of the eigenvalues of the Jacobian matrices cross the unit circle through the value -1 , see figure (15). Moreover, the $2 \mathrm{~T}$-periodic mode settles down after the destabilization of $\chi^{*}$. Due to the duty cycle saturation (see expressions (10) and (11)), two borderlines split the state plan into three regions defined as:

$$
\begin{gathered}
R_{0}:\left\{\chi_{i_{L}}[n] \mid \text { sat }\left(d_{k}[n]\right)=0\right\} \\
R_{1}:\left\{\chi_{i_{L}}[n] \mid \text { sat }\left(d_{k}[n]\right)=1\right\} \\
R_{2}:\left\{\chi_{i_{L}}[n] \mid \text { sat }\left(d_{k}[n]\right)=d_{k}[n]\right\}
\end{gathered}
$$




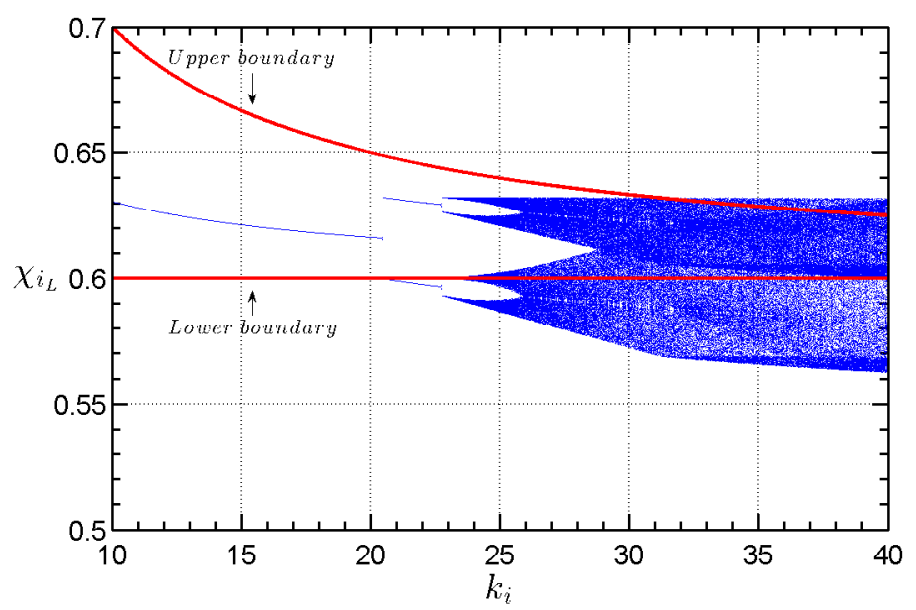

Figure 21: Bifurcation diagram of $\chi_{i_{L}}$ with respect to $k_{i}$ under action of proportional controller $\left(k_{v}=18.1\right)$.

A specific map models the photovoltaic system in each region.

When the current gain $k_{i}$ decreases until the critical value 20.8 , the $2 \mathrm{~T}$ periodic cycle hits the saturation boundary at 0, then the resulting Flip bifurcation is non-regular, it is called a degenerate flip bifurcation. When $k_{i}$ increases, a second flip bifurcation occurs and leads to a $4 \mathrm{~T}$-periodic behavior. This unstable dynamic causes the apparition of four cyclical chaotic sets, which are merged into two cyclical chaotic sets as the gain $k_{i}$ increases.

It has to be pointed out that this stability study is valid only for $k_{i}$ below the bifurcation value $k_{i, c r i t}$. The detected bifurcation is a local bifurcation.

\section{Conclusion}

Photovoltaic plant components are intrinsically nonlinear. Additionally, the interconnection of these components results in a complex system. Sometimes, when parameters undergo change, nonlinearities will begin to have a noticeable influence on system behavior. A major issue in the analysis of photovoltaic plant is the modeling of the multitude of components that make such complex system. Analysis tools and techniques can only provide useful information if 
the model accurately reflects true system behavior. The work highlights photovoltaic system dynamic analysis. This paper has summarized the discussion and simulation results of our study about the stability analysis problem associated with a stand-alone photovoltaic plant.

In this work, an asymptotic modeling of the photovoltaic array was presented. Moreover, the photovoltaic plant map has been developed. Thus, a state-space averaging method was adopted. We also detailed the modeling procedure of the selected power stage which consist of the two-cell dc-dc buck converter. Before starting the procedure of dynamic investigation, we have proposed a nonlinear PWM controller based on state-space averaging method. This controller was simulated and its performance was shown to be satisfactory. Thus a discrete-time model of the photovoltaic plant was described.

The obtained model has been simulated numerically to predict behaviors of the photovoltaic system. Therefore, it was computed bifurcations diagrams for the photovoltaic system controlled by a proportional controller, taking as bifurcation parameters the current gain. We have also determined the stability domain of T-periodic running mode by analyzing the Jacobian matrices. This complete study together with an experimental confirmation of the results on the Center for Research in STIC (CReSTIC) platform, constitute one line of future work.

\section{References}

[1] M. Abdelmoula, S. Moughamir, B. Robert, Design and modeling of a standalone photovoltaic system, in: 2014 15th International Conference on Sciences and Techniques of Automatic Control and Computer Engineering (STA), 2014, pp. 825-834.

[2] M. Abdelmoula, S. Moughamir, B. Robert, Analysis and implementation of nonlinear implicit differential-algebraic equations solver: Application to a photovoltaic power system, in: 2016 13th International Multi-Conference on Systems, Signals Devices (SSD), 2016, pp. 735-741. 
[3] W. Xiao, W. Dunford, A. Capel, A novel modeling method for photovoltaic cells, in: Power Electronics Specialists Conference, 2004. PESC 04. 2004 IEEE 35th Annual, Vol. 3, 2004, pp. 1950-1956 Vol.3.

[4] V. Lo Brano, A. Orioli, G. Ciulla, On the experimental validation of an improved five-parameter model for silicon photovoltaic modules, Solar Energy Materials and Solar Cells 105 (2012) 27-39.

[5] W. De Soto, S. Klein, W. Beckman, Improvement and validation of a model for photovoltaic array performance, Solar Energy 80 (1) (2006) 78-88.

[6] F. Toledo, J. M. Blanes, A. Garrigós, J. A. Martínez, Analytical resolution of the electrical four-parameters model of a photovoltaic module using small perturbation around the operating point, Renewable Energy 43 (0) (2012) 83-89.

[7] V. Lo Brano, A. Orioli, G. Ciulla, A. Di Gangi, An improved five-parameter model for photovoltaic modules, Solar Energy Materials and Solar Cells 94 (8) (2010) 1358-1370.

[8] K. Ishaque, Z. Salam, H. Taheri, Simple, fast and accurate two-diode model for photovoltaic modules, Solar Energy Materials and Solar Cells 95 (2) (2011) 586-594.

[9] H. Tian, F. Mancilla-David, K. Ellis, E. Muljadi, P. Jenkins, A cell-tomodule-to-array detailed model for photovoltaic panels, Solar Energy 86 (9) (2012) 2695-2706.

[10] K. Ishaque, Z. Salam, A. Shamsudin, M. Amjad, A direct control based maximum power point tracking method for photovoltaic system under partial shading conditions using particle swarm optimization algorithm, Applied Energy 99 (2012) 414-422.

[11] M. Alonsogarcia, J. Ruiz, F. Chenlo, Experimental study of mismatch and shading effects in the - characteristic of a photovoltaic module, Solar Energy Materials and Solar Cells 90 (3) (2006) 329-340. 
[12] K. H. Edelmoser, F. A. Himmelstoss, Analysis of a new high-efficiency DC-to-AC inverter, IEEE Transactions on Power Electronics 14 (3) (1999) $454-460$.

[13] B. Robert, A. El Aroudi, Discrete time model of a multi-cell dc/dc converter: Non linear approach, Mathematics and Computers in Simulation 71 (4-6) (2006) 310-319.

[14] A. E. Aroudi, B. Robert, L. Martinez-Salamero, Modelling and analysis of multicell converters using discrete time models, in: 2006 IEEE International Symposium on Circuits and Systems, 2006. ISCAS 2006. Proceedings, 2006, pp. 4 pp. -2164.

[15] M. Feki, A. El Aroudi, B. G. M. Robert, L. Martínez-Salamero, Optimizing the dynamics of a two-cell DC-DC buck converter by time delayed feedback control, Communications in Nonlinear Science and Numerical Simulation 16 (11) (2011) 4349-4364.

[16] L. M. Tolbert, F. Z. Peng, Multilevel converters as a utility interface for renewable energy systems, in: IEEE Power Engineering Society Summer Meeting, 2000, Vol. 2, 2000, pp. 1271-1274 vol. 2.

[17] T. A. Meynard, H. Foch, P. Thomas, J. Courault, R. Jakob, M. Nahrstaedt, Multicell converters: basic concepts and industry applications, IEEE Transactions on Industrial Electronics 49 (5) (2002) 955-964.

[18] C. K. Tse, Complex Behavior of Switching Power Converters, CRC Press, 2003.

[19] M. Feki, A. E. Aroudi, B. G. M. Robert, N. Derbel, Control of a two-cell $\mathrm{dc} / \mathrm{dc}$ converter in presence of saturating duty cycle, in: Power Electronics and Motion Control Conference, 2008. EPE-PEMC 2008. 13th, 2008, pp. $2120-2125$.

[20] A. E. Aroudi, J. Pelaez, M. Feki, B. G. M. Robert, Stability analysis of two-cell Buck converter driven DC motor with a discrete-time closed loop, 
in: 6th International Multi-Conference on Systems, Signals and Devices, 2009. SSD '09, 2009, pp. 1-6.

[21] B. Robert, C. Robert, Border collision bifurcations in a one-dimensional piecewise smooth map for a PWM current-programmed H-bridge inverter, International Journal of Control 75 (16-17) (2002) 1356-1367.

[22] F. L. Luo, H. Ye, Small Signal Analysis of Energy Factor and Mathematical Modeling for Power DC ndash;DC Converters, IEEE Transactions on Power Electronics 22 (1) (2007) 69-79.

[23] F. L. Luo, H. Ye, M. H. Rashid, Digital Power Electronics and Applications, Academic Press, 2010, google-Books-ID: R44dbJu_cYgC.

[24] M. Abdelmoula, S. Moughamir, B. Robert, Chaos in A Stand-Alone Photovoltaic Plant, in: The 20th World Congress of the International Federation of Automatic Control (IFAC), 2017, pp. 7960-7964.

[25] M. R. D. Castro, B. G. M. Robert, C. Goeldel, Experimental chaos and fractals in a linear switched reluctance motor, in: Proceedings of 14th International Power Electronics and Motion Control Conference EPE-PEMC 2010, 2010, pp. T10-45-T10-49.

[26] A. H. Nayfeh, B. Balachandran, Applied Nonlinear Dynamics: Analytical, Computational and Experimental Methods, John Wiley \& Sons, 2008.

[27] H. D. I. Abarbanel, R. Brown, M. B. Kennel, Variation of Lyapunov exponents on a strange attractor, Journal of Nonlinear Science 1 (2) (1991) 175-199.

[28] A. Wolf, J. B. Swift, H. L. Swinney, J. A. Vastano, Determining Lyapunov exponents from a time series, Physica D: Nonlinear Phenomena 16 (3) (1985) 285-317.

[29] G. Benettin, L. Galgani, A. Giorgilli, J.-M. Strelcyn, Lyapunov Characteristic Exponents for smooth dynamical systems and for hamiltonian systems; 
a method for computing all of them. Part 1: Theory, Meccanica 15 (1) (1980) 9-20.

[30] K. Geist, U. Parlitz, W. Lauterborn, Comparison of different methods for computing Lyapunov exponents, Progress of Theoretical Physics 83 (5) (1990) 875-893.

[31] H. F. von Bremen, F. E. Udwadia, W. Proskurowski, An efficient QR based method for the computation of Lyapunov exponents, Physica D: Nonlinear Phenomena 101 (1) (1997) 1-16.

[32] M. De Castro, B. Robert, C. Goeldel, Analysis of aperiodic and chaotic motions in a switched reluctance linear motor, in: Proceedings of 2010 IEEE International Symposium on Circuits and Systems (ISCAS), 2010, pp. 137-140.

[33] M. Abdelmoula, S. Moughamir, S. Martinez, B. Robert, Stability analysis of a photovoltaic power system, in: 2017 International Conference on Green Energy Conversion Systems (GECS), 2017, pp. 1-8. 